hep-th/0502211

CALT-68-2543

HUTP-05/A005

ITFA-2005-05

\title{
Hartle-Hawking Wave-Function for Flux Compactifications: The Entropic Principle
}

\author{
Hirosi Ooguri, ${ }^{1}$ Cumrun Vafa, ${ }^{2}$ and Erik Verlinde ${ }^{3}$ \\ 1 California Institute of Technology 452-48, Pasadena, CA 91125, USA \\ 2 Jefferson Physical Laboratory, Harvard University, Cambridge, MA 02138, USA \\ 3 Institute for Theoretical Physics, University of Amsterdam, \\ Valckenierstraat 65, 1018 XE Amsterdam, The Netherlands
}

\begin{abstract}
We argue that the topological string partition function, which has been known to correspond to a wave-function, can be interpreted as an exact "wave-function of the universe" in the mini-superspace sector of physical superstring theory. This realizes the idea of Hartle and Hawking in the context of string theory, including all loop quantum corrections. The mini-superspace approximation is justified as an exact description of BPS quantities. Moreover this proposal leads to a conceptual explanation of the recent observation that the black hole entropy is the square of the topological string wave-function. This wave-function can be interpreted in the context of flux compactification of all spatial dimensions as providing a physical probability distribution on the moduli space of string compactification. Euclidean time is realized holographically in this setup.
\end{abstract}

February, 2005 


\section{Introduction}

In string compactifications we typically start by assuming that the universe includes a non-compact macroscopic spacetime. Even though this is a natural assumption to make in the present epoch of cosmology, it is far less clear whether it is a good assumption in the very early universe. In particular one can imagine a universe arising from a microscopic/Planckian size compact space. In fact this seems like a more reasonable assumption in the context of cosmology.

On the other hand if we consider complete compactification of all spatial directions in string theory, we should not be able to freeze the moduli of string theory. More precisely we would expect to have a wave-function on the moduli space of string compactifications, whose amplitudes is peaked at the values of moduli where the classical equations of motion would be obeyed. Such a notion of wave-function seems necessary if one is to have a physical framework to compare the relative probabilities of different string compactifications.

Appealing as this idea is, there has been almost no progress in this direction in string theory. Our main aim in this paper is to present a modest progress in this direction for compactifications which preserve supersymmetry, where exact computations are possible. As a by-product, we are also able to make contact with the recent result that the exact black hole entropy is given by the norm square of the topological string partition function [1]. This connection arises since we are able to identify the partition function as the wave-function of the universe in the mini-superspace description, analogous to the HartleHawking construction [2]. The mini-superspace approximation is justified as an exact description of BPS quantities. Moreover we find the precise form of this wave-function which includes corrections to all orders in the string perturbation theory.

We believe this work is a first step toward developing a more realistic quantum cosmology within string theory. Given the recent exciting observational data, such a development would be rather timely.

The original Hartle-Hawking no-boundary proposal gives a construction of a special solution to the Wheeler-De Witt (WDW) equation to the Einstein gravity with a positive cosmological constant on a three-sphere $S^{3}$ in terms of a formal Euclidean functional integral over a four-dimensional ball $B_{4}$, which is bounded by the $S^{3}$. There are other fourdimensional spaces bounded by $S^{3}$, and they would give different solutions to the WDW equation. Hartle and Hawking proposed that the particular solution associated to $B_{4}$ plays a distinguished role as a wave function of the universe. This wave-function was used to set 
the initial boundary condition of the universe, which would expand exponentially due to the positive cosmological constant. Since the Euclidean functional integral in the Einstein gravity is not well-defined by itself, Hartle and Hawking considered a simplified system where the degrees of freedom are reduced to zero modes on $S^{3}$ - this is called the minisuperspace approximation. Their construction of the wave-function can be made more rigorous if the computation can be formulated in the string theory, where a full quantum theory of gravity is available.

In this paper, we will find a string theoretical realization of a similar construction for certain flux compactifications, where the cosmological constant is negativel Motivated by the recent result on the entropy of Calabi-Yau black holes [1], we are particularly interested in compactification of type II string on $S^{2}$ times a Calabi-Yau three-fold $M$ with fluxes so that we have a $(1+1)$-dimensional spacetime with a negative cosmological constant. To relate it to the black hole computation, we will further compactify the remaining one spatial dimension to a circle $S^{1}$ with the periodic (supersymmetry preserving) boundary condition on the fermions. To apply the Hartle-Hawking prescription, we look for a space that is bounded by $S^{1} \times S^{2} \times M$ and that supports a classical solution to the string equations of motion with an Euclidean signature metric. In the following, we will construct such a space and show that the topological string partition function gives the Hartle-Hawking type wave-function associated to this geometry. The black hole microstates are holographically dual to this geometry by exchange of a spatial and temporal direction in Euclidean space (target space analog of open/closed channel duality on the worldsheet). In particular the Euclidean gauge theory describing the black hole microstates leads to a holographic realization of Euclidean time in this setup. We also discuss some possible generalizations to other compactifications of string theory.

The organization of this paper is as follows: In section 2 we recast the black hole attractor mechanism as the extremization of flux generated superpotential upon compactifications on $S^{2} \times M$. In section 3 we introduce the notion of the mini-superspace for the problem at hand. In section 4 we review attractor flows in the context of black hole fluxes. In section 5 we setup the formulation of WDW equation in the BPS subsector of the mini-superspace. Here we propose a semi-classical notion of the wave function. In section 6 we make our proposal for the full wave function, where we make contact with

1 The Hartle-Hawking wave function for flux compactifications in de Sitter space was discussed in [3, 4 . 
the result of [1], in relating the black hole entropy to topological string wave-function. In section 7 we briefly consider more general compactifications. We conclude this paper with a discussion of some directions for future research in section 8 .

\section{Flux Compactifications and the Attractor Mechanism}

In this section we discuss attractor mechanism for black holes [5, 6] in a way to make contact with some of the recent discussions on compactifications of string theory [7]. The most thoroughly studied class involves compactifications on Calabi-Yau manifolds with fluxes turned on, similar to what we will be considering in the context of black holes. However, it is useful to start not from the black hole perspective, but from the perspective of compactification to $(1+1)$ dimensions.

\subsection{Compactification on Calabi-Yau times $S^{2}$}

Suppose we consider the compactification of type IIB superstrings to two dimensions on a Calabi-Yau threefold $M$ times a 2-sphere $M \times S^{2}$. Turn on 5 -form fluxes for the RR 5 -form field strength to be

$$
F_{5}=F_{3} \wedge \omega
$$

where $\omega$ is a unit volume form on $S^{2}$, and $F_{3}$ is a 3 -form on $M$. Choosing an integral basis of magnetic/electric $H^{3}(M)$ as $\left\{\alpha_{I}, \beta^{J}\right\}_{I=0, \ldots, h^{2,1}}$, we write

$$
F_{3}=\sum_{I}\left(p^{I} \alpha_{I}+q_{I} \beta^{I}\right)
$$

It is possible, as in [8], to write down a superpotential whose extremization leads to the condition for $(2,2)$ supersymmetry in $d=2$ :

$$
W=\int_{M \times S^{2}} F_{5} \wedge \Omega,
$$

where $\Omega$ is the holomorphic 3 -form on the Calabi-Yau three-fold. To deduce this superpotential, note that this superpotential is consistent with the tension (BPS mass in 1 dimension) of the domain wall D3 brane which wraps a 3-cycle in the Calabi-Yau and changes the $F_{5}$ flux.

The condition for extremization of $W$ and preserving supersymmetry is

$$
D W=0 .
$$


Geometrically it is the following variational problem: the complex structure of the CalabiYau are field variables and we can look for extrema of $W$ with respect to their variation. Denote variation of $\Omega$ in arbitrary direction of $H^{2,1}(X)$ by $\delta \Omega$. The supersymmetry condition (2.4) is equivalent to

$$
\int_{M \times S^{2}} \delta \Omega \wedge F_{5}=\int_{M} \delta \Omega \wedge F_{3}=0
$$

Since $F_{3}$ is also real, this implies thate $F_{3} \in H^{3,0}+H^{0,3}$. Using the fact that there is only one element in $H^{3,0}$ represented by $\Omega$, and using the reality condition for $F_{3}$ we deduce that

$$
F_{3}=\operatorname{Re}(C \Omega)
$$

for some complex number $C$. In other words

$$
p^{I}=\operatorname{Re}\left(C X^{I}\right), \quad q_{I}=\operatorname{Re}\left(C F_{I}\right)
$$

where

$$
X^{I}=\int_{A_{I}} \Omega, \quad F_{I}=\int_{B^{I}} \Omega
$$

and $\left(A_{I}, B^{I}\right)$ are 3 -cycles on $M$ that are dual to the 3 -forms $\left(\alpha_{I}, \beta^{I}\right)$. With the complex structure of the Calabi-Yau satisfying (2.5), supersymmetry is preserved with a suitable choice of metric in 2 spacetime dimensions, i.e. the $A d S_{2}$ metric. For such a complex structure, the superpotential $W$ is not zero, but it is proportional to $\int_{M} \Omega \wedge \bar{\Omega}$. In addition, the size of $S^{2}$ is also determined by the supersymmetry condition, and we find that

$$
\operatorname{Area}\left(S^{2}\right)=\pi C \bar{C} \int_{M} \Omega \wedge \bar{\Omega}
$$

In the following, we will set the constant $C$ to be equal to 1 with a suitable rescaling of $\Omega$.

This is exactly the content of the attractor mechanism. To case it in the standard description of the black hole attractor, consider D3 branes wrapping $q_{I}$ times on $A_{I}$ and $p^{I}$ times on $B^{I}$. This gives rise to a supersymmetric black hole in four dimensions, whose BPS mass $M_{B P S}$ is given by

$$
M_{B P S}^{2}=K^{-1}|W|^{2}
$$

2 This extremization condition is similar to the one encountered in a different context [9]. 
where the exponentiated Kähler potential $K$ is given by

$$
K=i\left(\bar{X}^{I} F_{I}-X^{I} \bar{F}_{I}\right)=-2 \operatorname{Im} \tau_{I J} X^{I} \bar{X}^{J}
$$

and $W$ is the superpotential (2.3), which can also be expressed as

$$
\begin{aligned}
W & =q_{I} \int_{A_{I}} \Omega-p^{I} \int_{B^{I}} \Omega \\
& =q_{I} X^{I}-p^{I} F_{I} .
\end{aligned}
$$

Here and in the following, we use the Einstein convention and always sum over repeated indices. The period matrix $\tau_{I J}$ is defined by

$$
\tau_{I J}=\frac{\partial F_{J}}{\partial X^{I}}
$$

Extremizing $M_{B P S}$ with respect to the complex moduli of $X$, with the constraint that $K$ is constant, reproduces the attractor equations (2.5), where the constant $C$ is identified as a Lagrange multiplier for the constraint $K=$ const.

For the purpose of this paper, it is useful to describe the attractor mechanism using the action principle [10]. Variation of the action

$$
\begin{aligned}
S & =-\frac{\pi}{4}(K(X, \bar{X})+2 i W(X)-2 i \bar{W}(\bar{X})) \\
& =-\frac{\pi}{4}\left(\int \Omega \wedge \bar{\Omega}+\int(\Omega+\bar{\Omega}) \wedge F_{3}\right)
\end{aligned}
$$

in arbitrary directions of $H^{3}(M)$ reproduces the attractor equations (2.5). To see this, note that variation of $\Omega$ in the $H^{2,1}$ direction, i.e. $D W=0$, does not change the value of $K$ because $\delta K=\int \delta \Omega \wedge \bar{\Omega}=0$. Thus, such a variation can be regarded as a variation of the action $S$ where $K$ is fixed. On the other hand, varying $\Omega$ parallel to itself (i.e. in the $H^{3,0}$ direction) precisely leads to (2.5) in the gauge $C=1$. Another way to see this is to take a direct variation of $S$ with respect to $X$. The extremum of $S$ is given by

$$
X^{I}=\left(\frac{-i}{\operatorname{Im} \tau}\right)^{I J}\left(q_{J}-\bar{\tau}_{J K} p^{K}\right) .
$$

Note that this is still a non-linear equation since $\tau_{I J}$ in the right-hand side is evaluated at $X$ as in (2.10). Taking the real and imaginary parts of this equation, one reproduces the attractor equation (2.5) with $C=1$. For later convenience, we define $X_{p, q}^{I}$ by

$$
X_{p, q}^{I}=\left.\left(\frac{-i}{\operatorname{Im} \tau}\right)^{I J}\left(q_{J}-\bar{\tau}_{J K} p^{K}\right)\right|_{\text {attractor }},
$$


where the period matrix in the right-hand side is evaluated at the attractor point.

The action $S$ has a further nice feature: Not only it reproduces the attractor equation by extremization, its value at the extremum is the semi-classical answer for the entropy! One can see this since (2.11) is quadratic in $\Omega$ and its extremization gives

$$
S=\frac{\pi}{4} \int \Omega \wedge \bar{\Omega}_{\mid \text {attractor value }}=-\frac{\pi}{2} \operatorname{Im} \tau_{I J} X_{p, q}^{I} \bar{X}_{p, q}^{J} .
$$

Later in this paper we will be making contact in the semi-classical limit between this action and the overlap of topological string wave-functions. Note that the action depends not only on the complex structure moduli of the Calabi-Yau three-fold but also on the overall scaling factor $X^{I}$. We will identify physical degrees of freedom for this extra factor and how it combines with the complex structure moduli and described by the action (2.11).

\section{Further Compactification on $S^{1}$ and Mini-Superspace}

In the context of the black hole entropy counting, it is natural to consider a further compactification on $S^{1}$ with periodic (supersymmetry preserving) boundary conditions on the fermions around the circle. As we will see, this set-up naturally leads to computation of the Witten index for black hole microstates in the Euclidean setup. Consider type IIB superstring compactified on the Calabi-Yau 3-fold $M$ times $S^{2} \times S^{1}$. There is a natural Euclidean solution to the classical equations of motion which develops from this spacelike section. It is the geometry $M \times S^{2} \times H_{2} / \mathbf{Z}$, where $H_{2}$ is the hyperbolic disk, i.e. the Euclideanized $A d S_{2}$, with the metric

$$
d s^{2}=d \rho^{2}+e^{2 \rho} d \tau^{2}
$$

and the $\mathbf{Z}$ quotient periodically identifies $\tau \sim \tau+\beta$, as shown in figure 1. Here we regard $\tau$ as the spacelike coordinate around the compactified circle $S^{1}$, and $\rho$ is the Euclideanized time direction. This geometry is invariant under supersymmetry, which squares to become a translation along the $\tau$ direction.

There is another way to view this geometry. A more traditional way to think about the metric (3.1) is to view $\tau$ as an Euclideanized time. Since the $\tau$ direction is compactified on $S^{1}$ with the supersymmetry preserving boundary condition, the vacuum amplitude of the string theory in this geometry can be regarded as the Witten index $\operatorname{Tr}(-1)^{F} e^{-\beta H}$, where $H$ is the translation generator along the $\tau$ direction. The Witten index counts the 
degeneracy of ground states of the supersymmetric black hole discussed in the previous subsection, and it will play an important role in the following sections.
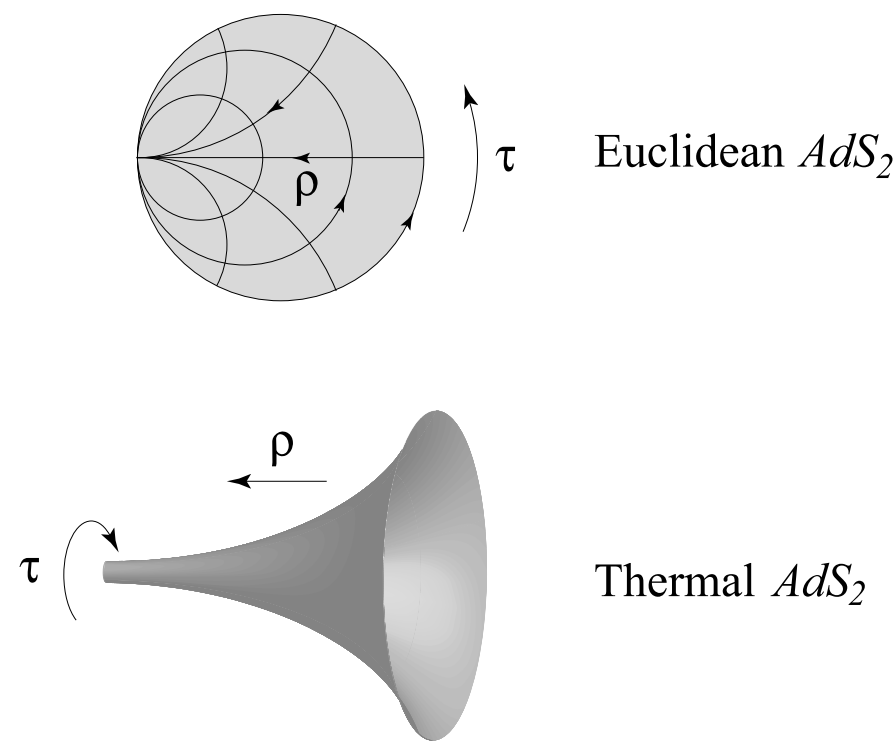

Thermal $\mathrm{AdS}_{2}$

Fig.1 Euclideanized $A d S_{2}$ is periodically identified in $\tau$ to make the thermal $A d S_{2}$ with the supersymmetry preserving boundary condition. We regard $\rho$ as an Euclidean time, which flows from $\rho=+\infty$ to $\rho=-\infty$.

On the other hand, if we view $\rho$ as an Euclideanized time, the geometry $M \times$ $S^{2} \times H_{2} / \mathbf{Z}$ describes an Euclidean time evolution of type II string compactified on $M \times S^{2} \times S^{1}$. This is how we were originally led to the metric (3.1). In this context, it is natural to consider wave-functions solving the WDW equation on $M \times S^{2} \times S^{1}$. Since the space is compact, the Calabi-Yau moduli are not fixed but rather fluctuate, and one should consider a wave function that is a function of these moduli.

The relation between these two points of view, depending on whether one regards $\tau$ or $\rho$ as the Euclideanized time in the target space, is analogous to the worldsheet duality relating an open string annulus diagram to an exchange of closed string states. The Witten index computation taking $\tau$ as the Euclideanized time direction is analogous to the computation in the open string channel, whereas the wave-function evolving in the $\rho$ direction is analogous to the boundary state in the closed string channel.

Let us consider a natural notion of a 'mini-superspace' from the second point of view, where we view $\rho$ as an Euclideanized time. Among relevant 
light modes are the complex moduli of the Calabi-Yau three-fold denoted by $z^{i}$ $\left(i=1, \ldots, h^{2,1}\right)$, which are in vector multiplets in four dimensions. The gravity multiplet also produces some scalar fields upon compactification on $S^{2} \times S^{1}$. One is the radius $R$ of $S^{2}$. Another scalar field is related to how the $S^{1}$ is fibered over $S^{2}$. More specifically, the compactification of the four-dimensional metric on $S^{1}$ produces a Kaluza-Klein gauge field in three dimensions, so we can consider its magnetic flux through the $S^{2}$. The additional scalar field $\varphi$ plays a role of the chemical potential for the flux. As we will see in section 5 , in the WDW equation, the radius $R$ and the chemical potential $\varphi$ naturally combine with the complex structure moduli $z$ of the Calabi-Yau to make a "large moduli space" with coordinates $X^{I}\left(I=0,1, \ldots, h^{2,1}\right)$. More explicitly, choose any holomorphic section $X_{0}^{I}(z)$ over the complex structure moduli space and define

$$
X^{I}=i 2 \operatorname{Re}^{i \varphi}\left(\frac{\bar{W}\left(X_{0}\right)}{K\left(X_{0}, \bar{X}_{0}\right) W\left(X_{0}\right)}\right)^{1 / 2} X_{0}^{I} .
$$

Note that the right-hand side is invariant under rescaling of $X_{0}^{I}$ and thus gives a well-defined function over the complex structure moduli space. It is instructive to further note that the exponentiated Kähler potential and the superpotential computed for $X^{I}$ have invariant meanings as,

$$
\begin{aligned}
& K(X, \bar{X})=-2 \operatorname{Im} \tau_{I J} X^{I} \bar{X}^{J}=(2 R)^{2}, \\
& W(X)=q_{I} X^{I}-p^{I} F_{I}=i 2 R e^{i \varphi} M_{B P S}(z, \bar{z}) .
\end{aligned}
$$

In four dimensions, there are several gauge fields $A^{I}\left(I=0, \ldots, h^{2,1}\right)$. One linear combination of these belongs to the gravity multiplet, i.e. it is the graviphoton, and the others are in the vector multiplets. Upon compactification on $S^{1}$, each gauge field becomes equivalent to a pair of massless scalar fields - one is the Wilson line of the gauge field along the $S^{1}$, and the other is the dual magnetic potential around the $S^{1}$,

$$
\phi^{I}=\oint_{S^{1}} A^{I}, \quad \tilde{\phi}_{I}=\oint_{S^{1}} \tilde{A}_{I},
$$

where $\tilde{A}_{I}$ is the dual of $A^{I}$ in four dimensions. One can also think of $\tilde{\phi}_{I}$ as the dual of the massless gauge field in three dimensions. By definition, they couple 
to the charges $\left(p^{I}, q_{I}\right)$ of the black hole (or the fluxe quantum numbers through $S^{2}$ in the near horizon geometry) as

$$
e^{i \sum_{I}\left(q_{I} \phi^{I}+p^{I} \tilde{\phi}_{I}\right)} .
$$

Combining with $X^{I}$ defined in (3.2), s set of four scalar fields $\left(X^{I}, \bar{X}^{I}, \phi^{I}, \tilde{\phi}_{I}\right)$ for each $I$ gives bosonic components of supermultiplets.

There are also hypermultiplet fields, which we denote collectively by $H$, which consist of the Kähler moduli of the Calabi-Yau together with the RRforms. We should also consider the radius $\beta$ of the $S^{1}$. A natural vacuum wave-function will then depend on these variables:

$$
\Phi_{p, q}\left(X^{I}, \bar{X}^{I} ; \phi^{I}, \tilde{\phi}_{I} ; H, \beta\right) .
$$

We also noted the dependence of the wave-function on the discrete choice of the 5 -form flux $F_{5}$ captured by the set of integers $\left(p^{I}, q_{J}\right)$.

For the vacuum state we would expect that the wave-function should not depend on the hypermultiplets $H$ or the radius $\beta$, at least to all orders in string perturbation theory. Since these moduli are not fixed by the classical equations of motion and also to all order in the perturbative expansion, it is natural to expect that the ground state wave-function does not depend on them.

The dependence of the wave-function on $\left(\phi^{I}, \tilde{\phi}_{I}\right)$ is also simple. Since $\phi^{I}$ is dual to $\tilde{A}_{I}$ and $\tilde{\phi}_{I}$ is dual to $A^{I}$ in three dimensions, we have

$$
\begin{aligned}
& G_{I J} \frac{d \phi^{J}}{d \rho}=\int_{S^{2}} \tilde{F}_{I}=q_{I}, \\
& G^{I J} \frac{d \tilde{\phi}_{J}}{d \rho}=\int_{S^{2}} F^{I}=p^{I},
\end{aligned}
$$

where $G_{I J}$ is the metric in the kinetic term for the gauge fields, and $F^{I}=d A^{I}$, $\tilde{F}_{I}=d \tilde{A}_{I}$. These equations means that, when we quantize the theory along the $\rho$ direction, $\left(\phi^{I}, \tilde{\phi}_{I}\right)$ are canonically conjugate to $\left(q_{I}, p^{I}\right)$. Therefore, the wave-function depends on $(\phi, \tilde{\phi})$ as

$$
\Phi_{p, q}(X, \bar{X} ; \phi, \tilde{\phi})=e^{i \sum_{I}\left(q_{I} \phi^{I}+p^{I} \tilde{\phi}_{I}\right)} \Psi_{p, q}(X, \bar{X}),
$$


if it is an eigenstate of the fluxe quantum numbers $(p, q)$. This $(\phi, \tilde{\phi})$ dependence is also expected from the fact that $(\phi, \tilde{\phi})$ are electric and magnetic static potentials for the black hole charges (3.4).

Thus, it is natural to look for a wave-function $\Psi$ which depends only on the $X^{I}$ and the amount of fluxes determined by $p, q$,

$$
\Psi_{p, q}(X, \bar{X})
$$

This wave-function should have the property that $\left|\Psi_{p, q}\right|^{2}$ is strongly peaked at the attractor value of the moduli (2.5) which is what extremizes the superpotential.

Do we know anything a priori about this mini-superspace wave-function? One question we may ask for such a wave-function is whether we can compare the different values of fluxes $(p, q)$ or if that is a super-selection sector. Since the dimensional reduction of the four-dimensional vector multiplets gives rise to the electric and magnetic potentials $\left(\phi^{I}, \tilde{\phi}_{I}\right)$ as dynamical variables and since they are conjugate to $(q, p)$, the wave-function $\Psi_{p, q}(X, \bar{X})$ should be defined over the entire landscape of vacua with different values of $(p, q)$. In fact, since we do not have any more spatial dimensions left, changing the fluxes does not cost infinite action and we should be able to compare different values $(p, q)$. This is not just a matter of principle, but in fact there are instantons that interpolate different amounts of fluxes. Such an instanton can be constructed as a domain wall D3 brane that wraps 3-cycles in the Calabi-Yau three-fold and winds around the $S^{1}$. Its action is $|\delta W|$ times $\beta$, where $\delta W$ is the variation of the superpotential (2.3) corresponding to the change of the fluxes.

In such a situation, would it be reasonable to expect the probability of the wave-function for the different flux sectors to be the same, namely

$$
\int d X d \bar{X}\left|\Psi_{p, q}(X, \bar{X})\right|^{2}=1 ?
$$

This may sound like the most economical assumption, in that we would be weighing each discrete choice of flux vacua equally. However, there is a reason to think that this is not natural. Consider the original Hartle-Hawking wavefunction for a three-sphere $S^{3}$. The saddle point computation of the wavefunction on $S^{3}$ can be viewed as filling it with a 4-dimensional ball with the $S^{3}$ as its boundary, and this leads to the action $S_{E}$ in the Euclidean ball,

$$
S_{E} \sim-\frac{1}{\Lambda}
$$


where $\Lambda$ is the cosmological constant, and the mini-superspace wave-function behaves as

$$
\Psi \sim \exp \left(-\frac{1}{\Lambda}\right) .
$$

In the present context $\Lambda<0$, and $-\frac{1}{\Lambda} \sim \operatorname{Area}\left(S^{2}\right) \sim S_{\text {entropy }}$, so we may expect that

$$
\int d X d \bar{X}\left|\Psi_{p, q}(X, \bar{X})\right|^{2} \sim \exp \left(S_{\text {entropy }}\right),
$$

namely the wave-function is normalized by the exponential of the entropy. It is natural since the string partition function on the full space $M \times S^{2} \times H_{2} / \mathbf{Z}$ should give the black hole entropy. Thus, at least semi-classically we expect (3.7) to hold.

In view of our discussion following (2.11) a natural guess for the probability measure is

$$
\left|\Psi_{p, q}(X, \bar{X})\right|^{2} \sim \exp \left[-\frac{\pi}{4} K-\frac{\pi}{2} i(W-\bar{W})\right]
$$

where $W(X)$ depends on the flux determined by $(p, q)$ as in (2.9). Indeed, the right-hand side is peaked at the attractor value and its value is given by the exponential of the entropy. To see this in terms of the physical variables, the complex structure moduli $z^{i}$ and the radius $R$ of the $S^{2}$, we can substitute (3.2) into (3.8) and find

$$
\left|\Psi_{p, q}\right|^{2} \sim \exp \left[-\pi\left(R^{2}-M_{B P S} R\right)\right]
$$

Extremizing this with respect to the complex structure moduli $z^{i}$ gives the attractor equation as we discussed in section 2.1 and extremizing with respect to $R$ gives

$$
\left|\Psi_{p, q}\right|_{\mid \text {extremum }}^{2} \sim \exp \left(\frac{\pi}{4} M_{B P S}^{2}\right)=\exp \left(S_{\text {entropy }}\right)
$$

reproducing the expected result.

In section 5, we will study a semi-classical function that solves the WDW equation. We will find (3.8) captures essential aspects of the wave-function. This same wave function will give a semi-classical approximation to the topological string partition function, which we will argue gives the exact answer for the Hartle-Hawking wave function including all string loop corrections. 
Note that (3.8) suggests that we can write the flux dependent part of the wave function as either a holomorphic function of $X$, given by

$$
\exp \left[-i \frac{\pi}{2} W_{p, q}(X)\right]
$$

(where we have written the subscripts of $(p, q)$ for $W$ to recall that $W$ depends on them), or in a real basis

$$
\exp \left[-i \frac{\pi}{4}\left(W_{p, q}(X)-\bar{W}_{p, q}(\bar{X})\right)\right]
$$

(A more accurate description of this will be given in sections 5 and 6.) In either case this suggests that 3 we should view the $p, q$ dependence of the state as obtained by an operator $O_{p, q}$ acting on the state corresponding to $p, q=0$

$$
\left|\Psi_{p, q}\right\rangle=O_{p, q}\left|\Psi_{0,0}\right\rangle
$$

Note that we also find that semi-classically $\Psi_{0,0}(X, \bar{X})=1$. It is a nice feature that this is an affine space in the sense that $O_{p, q} \cdot O_{p^{\prime}, q^{\prime}}=O_{p+p^{\prime}, q+q^{\prime}}$.

From this heuristic form of the wave function we see an extra unexpected structure which will play a key role in the following: The wave function is essentially a function of half of the variables (in the holomorphic description only a function of $X$ but not $\bar{X}$ ). As we will discuss below this is a reflection of the fact that we are considering the mini-superspace relevant for BPS quantities. This implies that on the reduced phase space of BPS quantities $X$ and $\bar{X}$ do not commute so that the wave function should have been only a function of half the variables. This also gives another reason why the wave-function does not depend on the hypermultiplet fields $H$ and the radius $\beta$ of $S^{1}$ as well as massive string fields. In order to show this we first turn to a discussion of attractor flow which is the equation of motion for the BPS quantities on the mini-superspace.

3 For this description, we should define $\Psi_{p, q}$ from a state $\Psi$ in a bigger Hilbert space including the choice of fluxes so that

$$
\langle p, q \mid \Psi\rangle\rangle=\left|\Psi_{p, q}\right\rangle
$$




\section{Attractor Flow Equations}

The wave-function $\Psi_{p, q}(X, \bar{X})$ should satisfy the Wheeler-De Witt equation. In mini-superspace the WDW equation corresponds to the quantization of the attractor flow for a black hole with charges $p^{I}$ and $q_{I}$. Therefore, in this section we review the equations that govern the classical attractor flow [5, 6, 11, 12]. The flow parameter will be identified with the Euclidean time $\rho$ in our setup.

Consider a ten-dimensional Euclidean metric of the form,

$$
d s^{2}=e^{2 U+2 \rho} d \tau^{2}+e^{-2 U} d \rho^{2}+e^{-2 U} d \Omega^{2}+d s_{\mathrm{CY}}^{2},
$$

where $\tau$ is the Euclideanized time direction compactified on $S^{1}, \rho$ is the radius coordinate, $d \Omega^{2}$ is the metric on a two-sphere of unit radius, and $d s_{\mathrm{CY}}^{2}$ is the metric on the internal Calabi-Yau three-fold. Note that $e^{-U}$ is the radius of the $S^{2}$, and the $A d S_{2}$ geometry is realized when $U$ is constant. Since we are interested in BPS configurations and since the supercharges preserved by the background square to become the translation along the $\tau$ direction, we assume that the scale factor $e^{U}$ and the complex moduli moduli $z^{i}\left(i=1, \ldots, h^{2,1}\right)$ of the Calabi-Yau three-fold are independent of $\tau$. In this case, we have a one-

dimensional system along the $\rho$ direction described by the effective action [11],

$$
\begin{aligned}
S_{\mathrm{eff}}=\frac{1}{2} \int_{-\infty}^{\infty} d \rho e^{\rho} & {\left[\left(\frac{d U}{d \rho}+1\right)^{2}+g_{i \bar{j}} \frac{d z^{i}}{d \rho} \frac{d \bar{z}^{\bar{j}}}{d \rho}\right.} \\
& \left.+e^{2 U}\left(M_{B P S}^{2}(z, \bar{z})+4 g^{i \bar{j}} \partial_{i} M_{B P S} \bar{\partial}_{\bar{j}} M_{B P S}\right)\right],
\end{aligned}
$$

where

$$
M_{B P S}=\sqrt{\frac{W(z) \bar{W}(\bar{z})}{K(z, \bar{z})}} .
$$

We regard $\rho$ as the Euclidean time of the system, which flows from $\rho=+\infty$ to $-\infty$. Since the effective action (4.2) can be written as

$$
\begin{aligned}
S_{\mathrm{eff}}=\frac{1}{2} \int_{-\infty}^{\infty} d \rho e^{\rho}[ & \left(\frac{d U}{d \rho}+1-e^{U} M_{B P S}\right)^{2}+ \\
& \left.+g_{i \bar{j}}\left(\frac{d z^{i}}{d \rho}-2 e^{U} g^{i \bar{m}} \bar{\partial}_{\bar{m}} M_{B P S}\right)\left(\frac{d \bar{z}^{\bar{j}}}{d \rho}-2 e^{U} g^{\bar{j} n} \partial_{n} M_{B P S}\right)\right] \\
& +(\text { total derivative }),
\end{aligned}
$$


the BPS equations are

$$
\begin{aligned}
& \frac{d U}{d \rho}=-1+e^{U} M_{B P S}(z, \bar{z}), \\
& \frac{d z^{i}}{d \rho}=2 e^{U} g^{i \bar{j}} \bar{\partial}_{\bar{j}} M_{B P S}(z, \bar{z}) .
\end{aligned}
$$

The signs on the right-hand side of these equations are chosen so that they are compatible with the initial condition at $\rho \rightarrow \infty$, which we regard as the infinite past in the Euclidean time.

The equations (4.4) can be combined into a single equation on the large moduli space. To write down such an equation, we start with a holomorphic section $X_{0}^{I}(z)\left(I=0,1, \ldots, h^{2,1}\right)$ over the moduli space of complex structure. They make projective coordinates of the moduli space, and as such there is a freedom to rescale these coordinates. We define the exponentiated Kähler potential $K_{0}$ and the superpotential $W_{0}$ for these coordinates as

$$
\begin{aligned}
& K_{0}=-2 \operatorname{Im} \tau_{I J} X_{0}^{I} \bar{X}_{0}^{J}, \\
& W_{0}=q_{I} X_{0}^{I}-p^{I} F_{I}\left(X_{0}\right) .
\end{aligned}
$$

We then combine the scale factor $e^{U}$ in the metric (4.1) and the complex moduli $z^{i}$ into a single set of coordinates $X^{I}$ defined by

$$
X^{I}=2 i e^{-U}\left(\frac{\bar{W}_{0}}{K_{0} W_{0}}\right)^{1 / 2} X_{0}^{I} .
$$

(This is the same as (3.2) with the identification that $e^{-U}$ is the radius of the $S^{2}$. Note that we are setting $\varphi=0$ since the $S^{1}$ is trivially fibered over $S^{2}$ in (4.1).) Note that the right-hand side of (4.5) is invariant under rescaling of $X_{0}^{I}$. Moreover

$$
K(X, \bar{X})=-2 \operatorname{Im} \tau_{I J} X^{I} \bar{X}^{J}=\left(2 e^{-U}\right)^{2}
$$

is the diameter squared of the $S^{2}$. Thus, the large moduli space parametrized by $X^{I}$ combines the complex moduli $z^{i}$ and the radius of the $S^{2}$, as we suggested earlier in section 3. Using $X^{I}$, the attractor flow equations (4.4) can be written as a single equation

$$
\frac{d X^{I}}{d \rho}=X^{I}+\left(\frac{i}{\operatorname{Im} \tau}\right)^{I J} \bar{\partial}_{J} \bar{W}(\bar{X}) .
$$


Let us show that (4.6) is equivalent to (4.4). If we multiply $\bar{X}^{J} \operatorname{Im} \tau_{J I}$ to both sides of (4.6), the left-hand side becomes

$$
\begin{aligned}
& \bar{X}^{I} \operatorname{Im} \tau_{I J} \frac{d X^{J}}{d \rho} \\
& =2 e^{-2 U} \frac{d U}{d \rho}-\frac{e^{-2 U}}{M_{B P S}}\left(\frac{d z^{i}}{d \rho} \partial_{i} M_{B P S}-\frac{d \bar{z}^{\bar{i}}}{d \rho} \bar{\partial}_{\bar{i}} M_{B P S}\right) .
\end{aligned}
$$

On the other hand, the right-hand side becomes

$$
\begin{aligned}
X^{I} \operatorname{Im} \tau_{I J} \bar{X}^{J}+i \bar{X}^{I} \partial_{I} \bar{W}\left(\bar{X}_{0}\right) & =-\frac{1}{2} K(X, \bar{X})+i \bar{W}(\bar{X}) \\
& =-2 e^{-2 U}+2 e^{-U} M_{B P S}
\end{aligned}
$$

Combining them together, we obtain

$$
\frac{d U}{d \rho}-\frac{1}{2 M_{B P S}}\left(\frac{d z^{i}}{d \rho} \partial_{i} M_{B P S}-\frac{d \bar{z}^{\bar{i}}}{d \rho} \bar{\partial}_{\bar{i}} M_{B P S}\right)=-1+e^{U} M_{B P S}
$$

The real part of this equation is precisely the first of (4.4):

$$
\frac{d U}{d \rho}=-1+e^{U} M_{B P S}
$$

The imaginary part gives

$$
\frac{d z^{i}}{d \rho} \partial_{i} M_{B P S}=\frac{d \bar{z}^{\bar{i}}}{d \rho} \bar{\partial}_{\bar{i}} M_{B P S}
$$

Similarly multiplying $\partial_{i} X_{0}^{J} \operatorname{Im} \tau_{I J}$ to both sides of (4.6) and using (4.7) and (4.8), we find

$$
\frac{d z^{i}}{d \rho}=2 e^{U} g^{i \bar{j}} \bar{\partial}_{\bar{j}} M_{B P S}
$$

Moreover, (4.9) implies (4.8) since both sides of (4.8) are now equal to $-2 e^{U} g^{i \bar{j}} \partial_{i} M_{B P S} \bar{\partial}_{\bar{j}} M_{B P S}$. Therefore, (4.6) for $X^{I}$ defined by (4.5) is equivalent to the standard BPS equations (4.4).

In (4.6), a general BPS solution can be easily expressed. Taking the real and imaginary parts of this equation, one finds

$$
\operatorname{Re}\left(X^{I}-\frac{d X^{I}}{d \rho}\right)=p^{I}, \quad \operatorname{Re}\left(F_{I}-\frac{d F_{I}}{d \rho}\right)=q_{I} .
$$


A general solution to this is then

$$
\begin{aligned}
& \operatorname{Re} X^{I}=p^{I}+c^{I} e^{\rho}, \\
& \operatorname{Re} F_{I}=q_{I}+d_{I} e^{\rho},
\end{aligned}
$$

where $\left(c^{I}, d_{I}\right)$ are integration constants specified by the initial condition at the infinite past $\rho=\infty$. Whatever initial condition one chooses there, $X^{I}$ at the infinite future $\rho \rightarrow-\infty$ are fixed to be at the attractor value,

$$
\operatorname{Re}\left(X^{I}\right) \rightarrow p^{I}, \quad \operatorname{Re}\left(F_{I}\right) \rightarrow q_{I}
$$

It is useful to write the BPS equation (4.6) as

$$
\operatorname{Im}\left(\tau_{I J}\right) \frac{d X^{I}}{d \rho}=-\frac{1}{2} \bar{\partial}_{I} K+i \partial_{I} \bar{W}
$$

In the next section we will use this equation to obtain the supersymmetric version of the WDW equation. Namely, after quantization it gives the BPS condition that when imposed on the states implies the WDW equation. In order to do this it is useful to keep in mind that the above BPS condition (4.10) can be derived from a local action with 4 supercharges of the form

$$
\int d^{4} \theta K+\left[\int d^{2} \theta W+\text { c.c. }\right] .
$$

The fact that the derivatives are replaced by covariant derivatives in (4.10) simply reflects the fact that we are discussing a local (gravitational) theory with 4 supercharges. This will be used in the next section to determine the commutation relation of fields restricted to the BPS sector.

\section{Wheeler-De Witt Equation and the Semi-Classical Wave Function}

In this section, we describe the semi-classical Hartle-Hawking type wave function for type II superstring associated to the spacelike geometry $M \times S^{2} \times S^{1}$. 


\subsection{Wheeler-De Witt Equation in Mini-Superspace}

We recall some basic aspects of WDW equation in the mini-superspace approach. The WDW equation is in general an infinite dimensional wave equation which takes the form,

$$
H|\Psi\rangle=0,
$$

where $|\Psi\rangle$ is a function of the superspace, which is the space of all possible metric data on the spacelike hypersurface, and $H$ is the Hamiltonian for the gravitational system. In the mini-superspace approach, $|\Psi\rangle$ becomes a wave function on a finite dimensional subspace of the superspace. In particular the above equation becomes similar to a ordinary wave equation for a quantum mechanical system. Most of the subtlety in solving such a reduced system is hidden in the choice of boundary conditions in solving this quantum mechanical system.

In the case at hand we have a stronger version of WDW equation, because we have supersymmetry. More precisely we are looking for a state $|\Psi\rangle$ which is supersymmetric,

$$
Q \mid \Psi>=0,
$$

with $Q^{2}=H$. Moreover, in this case, the reduction to the mini-superspace is not an approximation but is an exact description of the wave-function for computations involving BPS quantities.

The corresponding quantum mechanical system is a theory with 4 supercharges, with a superpotential $W=q_{I} X^{I}-p^{I} F_{I}$. This situation is a local (gravitational) version of the more familiar global (non-gravitational) LG theories with 4 supercharges, which we will now review, in order to gain insight into the case at hand. In particular we will consider the case of conformal theories where the corresponding superpotential $W\left(\Phi^{i}\right)$ is a quasi-homogeneous function of chiral fields $\Phi^{i}$.

- Global (Non-Gravitational) Case

This theory has been studied both in $(1+1)$ dimensions as well as in $(0+1)$ dimension, and as far as the vacuum geometry is concerned it is independent of the dimension, since the modes reduce to constant modes. As discussed in 
13] one can define a canonical notion of a distinguished vacuum state $|0\rangle$. This is done more naturally in the context of the $(1+1)$ dimensional theory where one consider the topologically twisted theory on a semi-infinite cigar, where the space is identified with the boundary circle. This gives a holomorphic basis of the vacuum. In particular $|0\rangle$ does not depend on the coupling constants of $\bar{W}$. With this choice of the vacuum state, the normalization of the vacuum state is unambiguous, and the norm

is well-defined. In fact, in the conformal case, it can be shown [13 that this overlap is given by

$$
\langle\overline{0} \mid 0\rangle=\int d \Phi d \bar{\Phi} \exp (-W(\Phi)+W(\bar{\Phi})) .
$$

Moreover there is a sense in which $\exp (-W(\Phi))$ is the wave function associated to $|0\rangle$ or more precisely in the same cohomology. In particular correlation functions of chiral operators evaluated in the vacuum are given by integrals involving $\exp (-W(\Phi))$ as

$$
\langle\overline{0}|f(\bar{\Phi}) g(\Phi)| 0\rangle=\int d \Phi d \bar{\Phi} f(\bar{\Phi}) g(\Phi) \exp (-W(\Phi)+W(\bar{\Phi}))
$$

Another way to state it is [13, 14]

$$
\langle\gamma \mid 0\rangle=\int_{\gamma} d \Phi \exp (-W(\Phi))
$$

where $\langle\gamma|$ is a boundary state defined by the Lagrangian D-brane related to $\gamma$. Given this, (5.1) follows since $|\gamma\rangle$ form a basis for ground states and we can use the Riemann bilinear identity to evaluate integrals.

A quick/heuristic way to see the relation between $|0\rangle$ and $\exp (-W(\Phi))$ is to note that since

$$
Q|0\rangle=0
$$

the supersymmetric flow conditions must be satisfied when evaluated on $|0\rangle$. The supersymmetric gradient flow is given by

$$
G_{i j} \frac{d \bar{\Phi}^{i}}{d \rho}-\frac{\partial W}{\partial \Phi_{j}}=0
$$


Upon quantization, we have

$$
G_{i j} \frac{d \bar{\Phi}^{i}}{d \rho} \rightarrow-\frac{\partial}{\partial \Phi_{j}}
$$

(note that we do not have $(-i)$ but $(-1)$ in the right-hand side since $\tau$ is the imaginary time) and the flow equation becomes

$$
\left(\frac{\partial}{\partial \Phi^{i}}+\frac{\partial W}{\partial \Phi^{i}}\right) \psi=0
$$

and one arrives at $\psi\left(\Phi^{i}\right) \sim \exp \left(-W\left(\Phi^{i}\right)\right)$. This is a rough derivation, and one could consult [13,14] for more precise statements.

\section{- Local (Gravitational) Case}

Now we turn to the case of interest for this paper. This differs in two important respects relative to what we just discussed. First of all, it is a local theory, involving gravity in addition to matter. A second difference is that the expression

$$
W(X)=q_{I} X^{I}-p^{I} F_{I}
$$

is only a leading computation of the superpotential. In particular one would expect that $F_{I}$ receives string quantum corrections as is suggested by the topological string amplitudes.

At first sight there is a third difference. Namely, in our situation the state $|\Psi\rangle$ is represented by a wave function $\Psi_{p, q}(X, \bar{X})$ on the full configuration space, and not (yet) by a holomorphic function $\psi_{p, q}(X)$. But, as we will explain below, this difference will disappear once we make use of the WDW equation. The supersymmetric WDW equation is equivalent to the quantum version of the BPS equation (4.6) and its complex conjugate. To understand the quantum version of the BPS equation, we note that the metric in the $X$ space implied by the effective action (4.2) is "almost" given by $\operatorname{Im}\left(\tau_{I J}\right)$ since

$$
\begin{aligned}
& X^{I} \operatorname{Im}\left(\tau_{I J}\right) \bar{X}^{J}=-2 R^{2}, \\
& D_{i} X^{I} \operatorname{Im}\left(\tau_{I J}\right) \bar{X}^{J}=X^{I} \operatorname{Im}\left(\tau_{I J}\right) \bar{D}_{\bar{j}} \bar{X}^{J}=0 \\
& D_{i} X^{I} \operatorname{Im}\left(\tau_{I J}\right) \bar{D}_{\bar{j}} \bar{X}^{J}=2 R^{2} g_{i \bar{j}},
\end{aligned}
$$


where

$$
D_{i} X^{I}=K \partial_{i}\left(K^{-1} X^{I}\right), \quad \bar{D}_{\bar{j}} \bar{X}^{J}=K \bar{\partial}_{\bar{j}}\left(K^{-1} \bar{X}^{J}\right),
$$

and $g_{i \bar{j}}=\partial_{i} \bar{\partial}_{\bar{j}} \ln K$. We note that $\operatorname{Im} \tau_{I J}$ has one negative sign in the direction of $H^{(3,0)}$ whereas it gives the standard positive definite metric in the $H^{(2,1)}$ direction. Flipping the sign in the $H^{(3,0)}$ direction gives what is denoted by $\operatorname{Im} \mathcal{N}_{I J}$ in the supergravity literature, which is the metric derived from the effective action (4.2). In the semi-classical approximation, flipping of the sign of the metric can be done by a suitable contour deformation in a functional integral, which we will freely do in this section. Thus, we will use $\operatorname{Im} \tau_{I J}$ as our metric and the corresponding quantization rule is

$$
\frac{\pi}{4} \operatorname{Im}\left(\tau_{I J}\right) \frac{d X^{J}}{d \rho} \rightarrow-\frac{\partial}{\partial \bar{X}_{J}} .
$$

(We did not derive the factor $\pi / 4$ in the right-hand side, but it was introduced in order for the quantum BPS equation to be consistent with the black hole entropy computation.)

Given this rule, the quantum version of the BPS equation (4.6) is

$$
\left(\frac{\partial}{\partial \bar{X}^{I}}-\frac{\pi}{8} \bar{\partial}_{I} K+i \frac{\pi}{4} \bar{\partial}_{I} \bar{W}\right) \Psi_{p, q}=0,
$$

and similarly for the complex conjugate equation

$$
\left(\frac{\partial}{\partial X^{I}}-\frac{\pi}{8} \partial_{I} K-i \frac{\pi}{4} \partial_{I} W\right) \Psi_{p, q}=0 .
$$

For future reference, let us denote the operator appearing in the constraint (5.4) $\bar{C}_{I}$ and the one in (5.5) by $C_{I}$, so that in terms of the state $\left|\Psi_{p, q}\right\rangle$ the constraints are $\bar{C}_{I}\left|\Psi_{p, q}\right\rangle=0$ and $C_{I}\left|\Psi_{p, q}\right\rangle=0$ respectively. Imposing both constraints is sufficient to determine the entire wave function. One finds in this way

$$
\Psi_{p, q}(X, \bar{X})=\exp \left[\frac{\pi}{8} K(X, \bar{X})+\frac{\pi}{4}(i W(X)-i \bar{W}(\bar{X}))\right] .
$$

One can use the BPS constraints also in a way that leads to a description of the covariant supersymmetric gradient flow, also known as the attractor flow, in terms of a holomorphic wave function $\psi_{p, q}(X)$. This wave function can be obtained by imposing first the constraint (5.4). This reduces $\Psi(X, \bar{X})$ 
essentially to a holomorphic function. The second condition (5.5) then fixes $\psi_{p, q}(X)$ to be given by

$$
\psi_{p, q}(X)=e^{i \frac{\pi}{2} W_{p, q}(X)}
$$

(See section 5.2 for further discussion on the holomorphic wave function.)

Needless to say this derivation is heuristic, but nevertheless, it is expected that at the semi-classical level $\psi_{p, q}$ should give a good approximation to the wave function. Note in particular that $\psi_{0,0}(X)=1$. In the remaining part of this paper we will provide evidence that this wave function coincides with the semi-classical approximation obtained from the topological string partition function, which we propose to be the exact Hartle-Hawking wave function including all string loop corrections.

\subsection{Reduced phase space, and the correspondence with topological strings}

There is an alternative way to view the result (5.6). Instead of imposing the quantum BPS condition (5.4), one can first solve the BPS condition (4.10) on the classical variables and then quantize the reduced system. The solutions of the first order BPS equations are determined by the initial values for $X^{I}$ and $\bar{X}^{I}$, and hence these parametrize the phase space of BPS configurations. In fact, the BPS equations can be viewed as second class constraints on the original phase space variables that include also the time derivatives $d X^{I} / d \rho$ and $d \bar{X}^{I} / d \rho$. The original Poisson brackets then change in to the Dirac brackets, and as a result $X^{I}$ and $\bar{X}^{I}$ become non-commuting variables.

We abbreviate the operators appearing in the constraints (5.4) and (5.5) by $\bar{C}_{I}$ and $C_{I}$ respectively, as mentioned above. We want to impose $\bar{C}_{I}$ on the ket state $|\Psi\rangle$ and its conjugate $\bar{C}_{J}^{\dagger}$ on the bra state $\langle\Psi|$. Notice that $\bar{C}_{J}^{\dagger}$ differs from $C_{J}$ in the sign of the derivative. The Dirac bracket is defined as

$$
\left[X^{I}, \bar{X}^{J}\right]_{\text {Dirac }}=\left[X^{I}, \bar{X}^{J}\right]-\sum_{K, L}\left[X^{I}, \bar{C}_{K}^{\dagger}\right] \frac{1}{\left[\bar{C}_{K}^{\dagger}, \bar{C}_{L}\right]}\left[\bar{C}_{L}, \bar{X}^{J}\right]
$$

where the denominator should be read as the inverse matrix. Here we only wrote commutators that we know are non-vanishing. For the constraints one finds

$$
\left[\bar{C}_{K}, \bar{C}_{L}^{\dagger}\right]=-\frac{\pi}{4} \bar{\partial}_{K} \partial_{L} K=\frac{\pi}{2} \operatorname{Im} \tau_{K L},
$$


while the commutators of the constraints with the coordinates give

$$
\left[X^{I}, \bar{C}_{K}^{\dagger}\right]=\delta_{K}^{I}, \quad\left[\bar{C}_{L}, \bar{X}^{J}\right]=\delta_{L}^{J}
$$

Inserting this in to the definition for the Dirac bracket leads to the following commutation relations for $X^{I}$ and $\bar{X}^{J}$

$$
\left[X^{I}, \bar{X}^{J}\right]_{\text {Dirac }}=\frac{2}{\pi}\left(\frac{1}{\operatorname{Im} \tau}\right)^{I J} .
$$

We like to stress that the non-commutative nature of the $X^{I}$ and $\bar{X}^{I}$ only arises because we are looking at the BPS configurations, and does not hold in the full theory. Physically what this means is that if we used the measuring devices made only of BPS objects we cannot simultaneously measure $X^{I}$ and $\bar{X}^{I}$. This situation is analogous to the quantization of two-dimensional electrons in a constant magnetic field, where the two-dimensional plane becomes non-commutative when the system is constrained to be on the first Landau level. Our derivation of the canonical commutation relation precisely mimics the analogous calculation for the Landau problem. The operators $\bar{C}$ and $\bar{C}^{\dagger}$ are like the creation/annihilation operators that appear in the Hamiltonian, while $C$ and $C^{\dagger}$ are the creation/annihilation operators that commute with the Hamiltonian and therefore act on the states in the lowest Landau level. On the constrained phase space the latter are linearly related to the coordinates $X$ and $\bar{X}$ on the original configuration space.

The commutation relations (5.7) imply that the symplectic form on the reduced phase space is given by the Kähler form derived from $K(X, \bar{X})$. In geometric quantization one starts from a phase space with a symplectic form and then one constructs the 'pre-quantum' Hilbert space of wave functions $\Psi(X, \bar{X})$. Next one picks a polarization and removes the dependence on half of the coordinates. For a Kähler manifold the natural polarization is to impose that wave functions are holomorphic. The Hilbert space for (5.7) can be thus be represented by holomorphic wave-functions $\psi\left(X^{I}\right)$ of $X^{I}$, with the inner product defined by

$$
\left\langle\psi_{1} \mid \psi_{2}\right\rangle=\int d X d \bar{X} e^{\frac{\pi}{4} K} \bar{\psi}_{1}(\bar{X}) \psi_{2}(X)
$$


Note that the weight factor $e^{\frac{\pi}{4} K}$ is determined so that the commutation relation (5.7) is compatible with the fact that $X^{I}$ and $\bar{X}^{J}$ are hermitian conjugate in the reduced Hilbert space. The constraint (5.4) is identical to the restriction imposed in Kähler quantization on the pre-quantum wave functions $\Psi(X, \bar{X})$ to get the quantum wave functions $\psi(X)$.

So far we have used the constraints $\bar{C}_{I}, \bar{C}_{I}^{\dagger}$. The other set of the constraints $C_{I}, C_{I}^{\dagger}$ determined the holomorphic wave-function as in (5.6). Its norm square according to the inner product $(5.8)$ is then

$$
\left\langle\psi_{p, q} \mid \psi_{p, q}\right\rangle=\int d X d \bar{X} \exp \left[\frac{\pi}{4} K(X, \bar{X})+\frac{\pi}{2} i(W(X)+W(\bar{X}))\right] .
$$

By performing the analytic continuation $(X, \bar{X}) \rightarrow(-X, \bar{X})$, this becomes

$$
\left\langle\psi_{p, q} \mid \psi_{p, q}\right\rangle=\int d X d \bar{X} \exp \left[-\frac{\pi}{4} K(X, \bar{X})-\frac{\pi}{2} i(W(X)-W(\bar{X}))\right] .
$$

This reproduces the semi-classical probability measure (3.8) we conjectured based on the entropy argument. After the analytic continuation, the holomorphic wave function becomes

$$
\psi_{p, q}(X)=e^{-i W(X)},
$$

which also agrees with what we expected in section 3 .

The Kähler quantization of the reduced phase space using the Kähler potential $K(X, \bar{X})$ is only possible in the semi-classical approximation. Due to the non-linearity of $K(X, \bar{X})$ there are many normal ordering problems that are hard if not impossible to solve. Fortunately, the quantization of the reduced phase space can be simplified by making an appropriate change of variables. Motivated by the form of the attractor equations, let us introduce real coordinates $\chi^{I}$ and $\eta_{I}$ via

$$
\chi^{I}=\operatorname{Re}\left(X^{I}\right) \quad \eta_{I}=\operatorname{Re}\left(F_{I}\right) .
$$

To determine the symplectic form for these new coordinates, let us calculate the commutator of the expressions on the right-hand side at the level of Poisson brackets, i.e. ignoring any operator ordering. One finds

$$
\begin{aligned}
{\left[\operatorname{Re}\left(X^{I}\right), \operatorname{Re}\left(F_{J}\right)\right]_{\text {Dirac }} } & =\frac{1}{4}\left[X^{I}, \bar{F}_{J}\right]_{\text {Dirac }}+\frac{1}{4}\left[\bar{X}^{I}, F_{J}\right]_{\text {Dirac }} \\
& =\frac{1}{2 \pi}\left(\frac{1}{\operatorname{Im} \tau}\right)^{I K}\left(\bar{\tau}_{K J}-\tau_{K J}\right) \\
& =\frac{1}{\pi i} \delta^{I}{ }^{I}
\end{aligned}
$$


and hence we conclude in the real $(\chi, \eta)$ coordinates the symplectic form is simply the canonical one leading to standard commutation relations

$$
\left[\chi^{I}, \eta_{J}\right]_{\text {Dirac }}=\frac{1}{\pi i} \delta_{J}^{I}
$$

The change of variables that we just described has a natural interpretation in terms of the space of close three forms $H^{3}(M, R)$. A three-form $\gamma \in H^{3}(M)$ can be parametrized in terms of the coordinates $\chi^{I}$ and $\eta_{J}$ as

$$
\gamma=\chi^{I} \alpha_{I}+\eta_{I} \beta^{I}
$$

in terms of the canonical basis $\alpha_{I}$ and $\beta^{J}$. The complex coordinates $X^{I}$ that are used in the WDW equation represent the periods of the (anti-)holomorphic three form $\Omega(X)$, which can be written in the canonical basis as

$$
\Omega(X)=X^{I} \alpha_{I}+\partial_{I} F_{0}(X) \beta^{I}
$$

The form of the canonical transformation (5.10) follows from by making the identification $\gamma=\operatorname{Re} \Omega$. The fact that $(\chi, \eta)$ are canonically conjugate variables also follows from the natural symplectic structure on $H^{3}$.

What we have achieved now is that the problem of quantizing the reduced phase space of BPS coordinates $X^{I}$ and $\bar{X}^{I}$ is mapped on to the much simpler problem of quantizing $H^{3}(M)$ using the real $(\chi, \eta)$-coordinates. Wave functions are in this case simply given as $\psi(\chi)$ with the familiar inner product. The price that one has to pay, however, is that wave function that are simple in terms of the $X^{I}$ variables will look complicated in terms of $\chi$. In particular, we argued that the holomorphic wave function $\psi_{0,0}(X)$ to be used in the solution of the WDW equation for $p=q=0$ is just $\psi_{0,0}(X)=1$. The corresponding wave function $\psi_{0,0}(\chi)$ in the real variables will not be as simple, in fact, we will show that it is equal to the topological string partition function!

The relation between the wave function in the real and complex polarization can be found at the semi-classical level by applying standard canonical transformation techniques (see e.g. [15]). In classical mechanics canonical transformations can be described with the help of a generating function. In our case this generating function should depend on one of the real and one of the complex coordinates. Since we are interested in transforming wave functions $\psi(X)$ 
to $\psi(\chi)$ the appropriate choice is to use a function $S(X, \chi)$ of the real coordinate $\chi^{I}$ and the complex coordinate $X^{I}$. It is determined by requiring that the canonical transformation (5.10) takes the form

$$
\eta_{I}=\frac{1}{i \pi} \frac{\partial S(X, \chi)}{\partial \chi^{I}}, \quad \bar{X}^{I}=\frac{2}{i \pi}\left(\frac{1}{\operatorname{Im} \tau}\right)^{I J} \frac{\partial S(X, \chi)}{\partial X^{J}} .
$$

After a little algebra one finds

$$
S(X, \chi)=\frac{i \pi}{2}\left(\chi^{I} F_{I}(X)-\frac{1}{2} F_{0}(X)+\frac{1}{2} \bar{F}_{0}(2 \chi-X)\right) .
$$

This leads to the equations

$$
\eta_{I}=\frac{1}{2} F_{I}(X)+\frac{1}{2} \bar{F}_{I}(2 \chi-X), \quad \bar{X}^{I}=2 \chi^{I}-X^{I},
$$

which are equivalent to (5.10). The relation between the semi-classical wave function $\psi(X)=e^{g(X)}$ and the corresponding wave function $\psi(\chi)=e^{f(\chi)}$ in the real polarization is

$$
\psi(X)=\int d \chi e^{\frac{i}{\pi} S(X, \chi)} \psi(\chi)
$$

where it is understood that the right-hand side is computed in the saddle point approximation. In terms of the functions $g(X)$ and $f(\chi)$ this gives the relation

$$
g(X)=f(\chi)+\frac{i}{\pi} S(X, \chi)
$$

where $\chi^{I}$ is solved in terms of $X^{I}$ through

$$
\frac{\partial f(\chi)}{\partial \chi^{J}}+\frac{i}{\pi} \frac{\partial S(X, \chi)}{\partial \chi^{J}}=0
$$

We want to determine the function $f(\chi)$ that corresponds to the simple wave function $\psi_{0,0}(X)=1$. In that case $g(X)=0$ and hence by differentiating (5.14) one gets

$$
\frac{\partial}{\partial X^{J}}\left(f(\chi(X))+\frac{i}{\pi} S(X, \chi(X))\right)=0,
$$

where $\chi(X)$ is solved from the condition (5.15). By inserting the expression (5.12) on the right-hand side one finds that this equation obeyed when $X^{I}=$ $2 \chi^{I}$. Putting this back in to (5.14) with $g(X)=0$ finally gives

$$
f(\chi)=\chi^{I} F_{I}(\chi)-F_{0}(\chi)=F_{0}(\chi),
$$

where we used homogeneity of $F_{0}(\chi)$. So we conclude that the canonical transformation maps the wave function $\psi_{0,0}(X)=1$ on to

$$
\psi_{0,0}(\chi)=e^{F_{0}(\chi)} .
$$

This is precisely the semi-classical approximation to the topological partition function! 


\section{Topological Strings and the Exact Hartle-Hawking Wave-function}

So far we have argued using semi-classical reasoning the form of the HartleHawking wave function. The aim of this section is to propose an exact such wave function which agrees in the semi-classical limit with the wave function discussed before. We will argue that the state with no flux can be identified with the topological string wave function:

$$
\left|\psi_{0,0}\right\rangle=\left|\psi_{\text {top }}\right\rangle
$$

That this relation could hold presupposes that topological string partition function also corresponds to a wave function associated to quantizing $H^{3}(M)$. This is indeed the interpretation of [16] of the holomorphic anomaly for topological strings [17]. Moreover this is in agreement with the fact that at least semi-classically the topological string partition function describes the HartleHawking wave function in the real polarization as discussed in the previous section. Moreover we argue that the semi-classical result

$$
\left|\psi_{p, q}\right\rangle=O_{p, q}\left|\psi_{0,0}\right\rangle
$$

also holds with the expected factor

$$
O_{p, q}=\exp \left[-\frac{i \pi}{4} W_{p, q}+\text { c.c. }\right]
$$

interpreted as an operator acting on the topological string Hilbert space. Important evidence for this proposal comes from the connection between the black hole entropy and the topological string partition function discovered in [1]. To motivate this more clearly let us first briefly review the result of [1].

Semi-classically, the entropy of the BPS black hole obtained by wrapping D3 branes around cycles of $M$ is given by the area (2.6) of the horizon. It was pointed out in [1], based on earlier work [18], that the resulting quantum corrected entropy formula can be concisely expressed as

$$
S_{\mathrm{BH}}(q, p)=\mathcal{F}(p, \phi)+\sum_{I} q_{I} \phi^{I}
$$

where

$$
\mathcal{F}(p, \phi)=F_{\text {top }}(X)+\bar{F}_{\text {top }}(\bar{X}), \quad X=p+\frac{i}{\pi} \phi
$$


and

$$
F_{\text {top }}(X)=\sum_{g=0}^{\infty} F_{g}(X)
$$

is the full topological string partition function.

Moreover the quantum corrected attractor equations also take the simple form:

$$
q_{I}=-\frac{\partial}{\partial \phi^{I}} \mathcal{F}(p, \phi)
$$

At the attractor point, the string perturbation expansion is an asymptotic expansion for large black hole charges. Since (6.2) takes the Legendre transformation from $\phi$ to $q$, it was conjectured in [1] that the number of states $\Omega(p, q)$ of the black hole with finite charges $p^{I}, q_{I}$ is given by Laplace transformation of the topological string partition function,

$$
\begin{aligned}
\Omega(p, q) & =\int d \phi e^{-q_{I} \phi^{I}+\mathcal{F}(p, \phi)} \\
& =\int d \phi e^{-q_{I} \phi^{I}}\left|e^{F_{\mathrm{top}}\left(p+\frac{i}{\pi} \phi\right)}\right|^{2},
\end{aligned}
$$

More precisely, the conjecture states that $\Omega(p, q)$ given by (6.5) is the Witten index for the quantum Hilbert space of the black hole. This conjecture has been successfully tested 1 in examples where both $\Omega(p, q)$ and $F_{\text {top }}(X)$ can be computed independently and explicitly [19, 20,21] 0 .

We note that the relation (6.5) is an example of the large $N$ duality. The near horizon limit in the above is equivalent to the low energy limit for the open string system on D3 branes wrapping 3-cycles on $M$ and extended in one time-dimension, and the entropy counts the number of ground states of this system (with the sign $(-1)^{F}$ ). The conjecture (6.5) states that this can also be evaluated using the closed topological string theory on $M$. In principle the gauge theory on the D3-brane provides a non-perturbative holographic definition of topological string theory.

4 In addition there are interesting non-perturbative contributions discovered in these papers which suggest how to define topological strings beyond perturbation theory.

5 See also [22]. It should be reminded that the expression [1] is proposed to be valid for the perturbative expansion, as stressed in [19,20]. Thus, the test of the conjecture [1] has to be made in the perturbative regime where the topological string coupling constant is small. 
As noted in [1] the expression (6.5) for the number of states can be written in a nice way as a Wigner function. Namely, by taking the contour of the $\phi$ integral in (6.5) along the imaginary axis as $\phi=-i \pi \chi$, one gets

$$
\Omega(p, q)=\int d \chi e^{i \pi q \chi} \bar{\psi}_{\mathrm{top}}(p-\chi) \psi_{\mathrm{top}}(p+\chi),
$$

where

$$
\psi_{\text {top }}(\chi)=e^{F_{\text {top }}(\chi)}
$$

is the exact topological string partition function.

To relate this result to the normalization of the Hartle-Hawking wave function, let us start with the following observation. The Witten index $\Omega(p, q)$ for the black hole can be evaluated by analytically continuing the near horizon geometry to $M \times S^{2} \times\left(\right.$ Euclidean $A d S_{2}$ ), by periodically identifying the Euclidean time $\tau$ by period $\beta$, and by computing the partition function with the supersymmetry preserving boundary condition around the $\beta$-circle. The result should be independent of $\beta$.
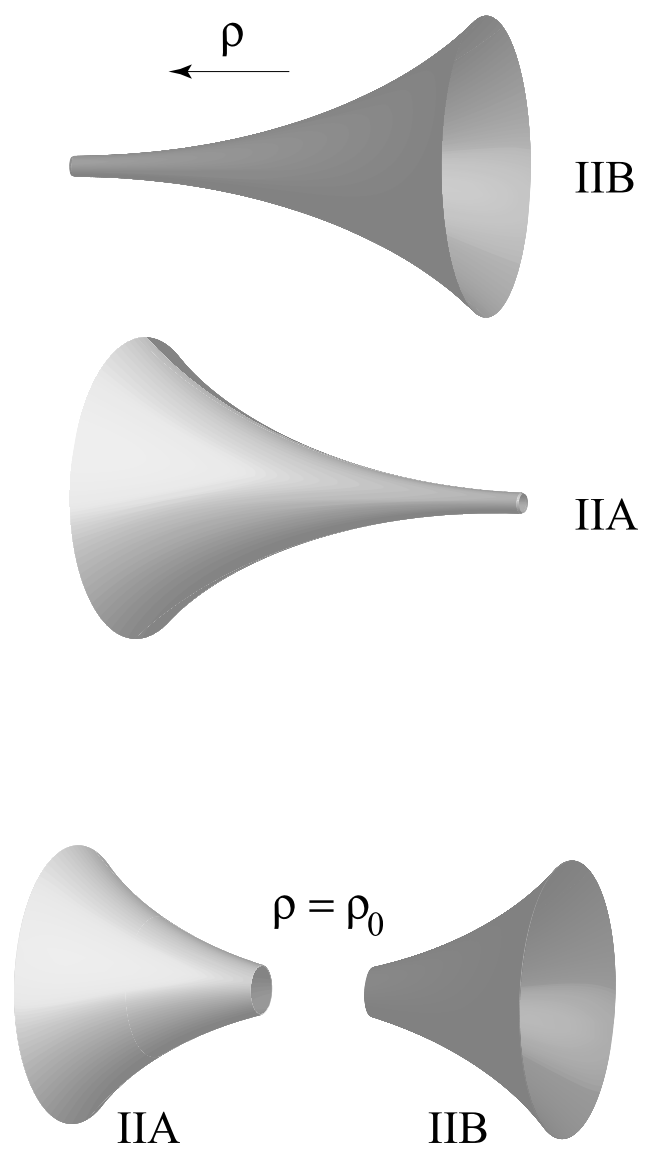
Fig.2 The periodically identified Euclidean $A d S_{2}$ and its T-dual are shown in the above. We can combine the right-hand half of the IIB picture and the left-half of the IIA picture so that the geometric description is valid at both sides.

The Euclidean $A d S_{2}$ with the periodic identification, i.e. $H_{2} / \mathbf{Z}$, has the topology of a cylinder. At one end the radius goes to infinity and at the other end it approaches zero. Now, separate the cylinder into two parts by cutting it across at $\rho=\rho_{0}$ for some fixed $\rho_{0}$. We want to associate a wave function with both sides. The side at which the radius goes to infinity satisfies the quantum BPS equation corresponding to the 'upward' attractor flow, while the other side at which the radius goes to zero is represented by a conjugate state corresponding to the 'downward' attractor flow.

One could argue that the geometric description of the solution for radii much smaller than string length is not trustable, and than should go to the T-dual description. In this case we end up with a Euclidean IIA theory where radius is increasing. On the original IIB side, we have the near horizon geometry of the D3 branes wrapping 3-cycles in the Calabi-Yau 3-fold and wrapping the $S^{1}$. On the IIA side, we have Euclidean D2 branes wrapping the same 3-cycles in the Calabi-Yau and smeared over the dual $S^{1}$. The mini-superspace wavefunctions on both sides are given by the topological string partition function since the geometric set-ups in the Calabi-Yau 3-fold are the same. Thus, viewed in this way, our Euclidean geometry is separated into two parts, the IIB part for $\rho>\rho_{0}$ and the IIA part for $\rho<\rho_{0}$, as shown in Figure 2. This is similar to the Euclidean $A d S_{2}$ periodically identified in the global time. The metric is given by (3.1) in the IIB side.

This naturally suggests an interpretation of the Witten index $\operatorname{Tr}(-1)^{F} e^{-\beta H}$ as an inner product in the Hilbert space for the space $M \times S^{2} \times S^{1}$. As we mentioned earlier, this is analogous to the familiar worldsheet duality in the perturbative open string theory where an annulus diagram can be represented either as a trace over an open string Hilbert space or an inner product of boundary states. The difference is that we are performing this in the target space. 
Let $\left|\psi_{p, q}\right\rangle$ denote the state we obtain upon doing the path-integral on the right in a fixed flux sector. The above consideration leads to the statement that

$$
\Omega(p, q)=\left\langle\psi_{p, q} \mid \psi_{p, q}\right\rangle
$$

To make contact between the discussion in section 5 and the topological string let us write the expression (6.5) in invariant form

$$
\Omega(p, q)=\left\langle\bar{\psi}_{\text {top }}\left|e^{i \pi(q \chi-p \eta)}\right| \psi_{\text {top }}\right\rangle
$$

where $\chi$ and $\eta$ are to be regarded as operators, and the state $\bar{\psi}$ is defined by the wave function $\psi^{*}(-\chi)$. Here, the state on the left is not simply the complex conjugate but it contains a minus sign due to the time reversal in the attractor flow equation. Next we note that the attractor relations (5.10) imply that $q_{I} \chi^{I}-p^{I} \eta_{I}=\operatorname{Re} W(X)$, and hence

$$
\Omega(p, q)=\left\langle\bar{\psi}_{\text {top }}\left|e^{\frac{-\pi}{2} i(W(X)-\bar{W}(\bar{X}))}\right| \psi_{\text {top }}\right\rangle
$$

It follows that if we identify

$$
\left|\psi_{p, q}\right\rangle=e^{-i \frac{\pi}{4}(W(X)-\bar{W}(\bar{X}))}\left|\psi_{\text {top }}\right\rangle
$$

or in the wave function form as

$$
\psi_{p, q}\left(\phi^{I}\right)=e^{-\frac{1}{2} q_{I} \phi^{I}+F_{\mathrm{top}}\left(p^{I}+\frac{i}{\pi} \phi^{I}\right)},
$$

then

$$
\Omega(p, q)=\left\langle\psi_{p, q} \mid \psi_{p, q}\right\rangle=\int d \phi^{I}\left|\psi_{p, q}\left(\phi^{I}\right)\right|^{2}
$$

exactly as expected. Moreover the form of the wave function (6.8) is exactly consistent with the semi-classical reasoning which led to (6.1). The fact that the wave functions for both the IIA and IIB side would lead to the same state is clear once we recall that the internal part of the Calabi-Yau and thus the mini-superspace is identical for both cases where a D2 brane IIA instanton is playing the role of D3 brane of IIB. We find this a highly non-trivial evidence for our conjecture for the exact Hartle-Hawking wave function.

Note that even though for many purposes the semi-classical wave function we presented earlier may suffice, the very fact that in a consistent quantum 
theory of gravity the exact quantum corrected Hartle-Hawking wave function could be made sense out of is a rather significant statement.

\section{Holomorphic versus Real Polarization}

We end this section with a brief comment on the issue of normal ordering in the Kähler quantization in terms of the $(X, \bar{X})$ coordinates and its connection to the holomorphic anomaly equations. We also make contact with the work in [23] which reformulates the black hole entropy proposal of [1] in a background independent way.

To give a precise definition of the Kähler quantization procedure discussed in section 5 one has to resolve the operator ordering ambiguities that arise due to the non-linearity of the canonical transformation (5.10) from $(\chi, \eta)$ to $(X, \bar{X})$. In the discussion above we have ignored this issue, so part of our arguments are semi-classical. In particular, the relation between the wave function $\psi_{\text {top }}(X)$ and the exact topological string partition function $\psi_{\text {top }}(\chi)$

$$
\psi_{\text {top }}(X)=\int d \chi e^{\frac{i}{\pi} S(X, \chi)} \psi_{\text {top }}(\chi)
$$

is a semi-classical formula. Nevertheless, the topological string is well defined and its partition function exists to all orders in perturbation theory. Hence, in principle it describes the loop corrections to the Hartle-Hawking wave function to all orders in perturbation theory.

The partition function of topological string theory can be computed perturbatively around a given background by writing $X^{I}=Z^{I}+x^{I}$, and treating

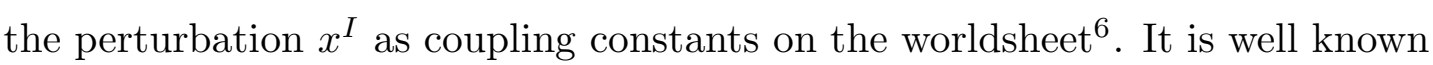
that the resulting partition function $\psi_{\text {top }}(x ; Z, \bar{Z})$ depends holomorphically on $x^{I}$ but has a non-holomorphic dependence on the background due to holomorphic anomaly. One of the original motivations for interpreting $\psi_{\text {top }}$ as a wave function is that it naturally explains this background dependence [16]. Namely, it arises because the topological string partition function is the wave function of the state $\left|\psi_{\text {top }}\right\rangle$ in a complex polarization that is determined by the background. In fact, the coordinates $x^{I}$ used by the perturbative topological string

6 The real polarization corresponds to setting the background $Z^{I}$ at infinity where the magnetic $B$ cycles are infinitely bigger than the electric $A$ cycles. 
are a linearization of the 'curved' $X^{I}$ coordinates. To be precise, the relation with $(\chi, \eta)$ is

$$
\chi^{I}=\operatorname{Re}\left(Z^{I}+x^{I}\right) \quad \eta_{I}=\operatorname{Re}\left(F_{I}(Z)+\tau_{I J} x^{J}\right),
$$

where $\tau_{I J}=\partial_{I} \partial_{J} F_{0}(Z)$ is determined by the background. These are just the attractor equations (5.10) linearized around $X^{I}=Z^{I}$. In this way the topological string avoids the normal ordering problems but at the cost of a background dependence. The partition function $\psi_{\text {top }}(x ; Z, \bar{Z})$ is related to the background independent wave function $\psi_{\text {top }}(\chi)$ via the Bargmann transform 24,23

$$
\psi_{\text {top }}(x ; Z, \bar{Z})=|\operatorname{det} \operatorname{Im} \tau|^{\frac{1}{2}} \int d \chi e^{i S(x, \chi ; Z, \bar{Z})} \psi_{\text {top }}(\chi)
$$

with

$$
S(x, \chi ; Z, \bar{Z})=\frac{\pi}{4} \bar{\tau}_{I J}(\bar{Z}) \chi^{I} \chi^{J}+\pi \chi_{I}\left(x^{I}+Z^{I}\right)+\frac{\pi}{4}\left(x^{I}+Z^{I}\right)\left(x_{I}+Z_{I}\right),
$$

where indices are lowered with $\operatorname{Im} \tau_{I J}(Z)$. This expression is a linearization of the generating function $S(X, \chi)$ defined in (5.12). The topological partition function $\psi(x ; Z, \bar{Z})$ thus gives in a certain sense a linearized description of the Hartle-Hawking wave function $\psi_{\text {top }}(X)$. We like to stress, however, that it is not a 'linear approximation': it is an exact wave function that carries the complete information about the state $\left|\psi_{\text {top }}\right\rangle$, and hence about the Hartle-Hawking wave function.

\section{More General Compactifications}

It is clear that the above philosophy, i.e. finding a wave function for string compactifications should be a generally valid principle when we compactify all dimensions. So in particular we should aim to write a wave function for the string vacuum upon compactification of type II superstrings on $X^{9}$ or for Mtheory on $X^{10}$. There are many interesting cases to consider, for example when $X^{9}=X^{6} \times S^{3}$ where $X^{6}$ is a Calabi-Yau and $S^{3}$ has some fluxes through it. One could also consider the case where $X^{9}=S^{5} \times S^{4}$; this may be relevant in the context of the usual $S^{5} \times A d S_{5}$ compactifications of type IIB. We leave a study of such wave functions to future work. Instead here we consider a 
particular case motivated by the example of 5 dimensional black holes obtained upon compactifications of M-theory on a Calabi-Yau threefold $X^{6}$.

Consider $M$-theory compactified on a spatial geometry $X^{6} \times S^{3} \times S^{1}$, where we turn on the 7 -form field strength flux $* G$ as

$$
* G=F_{4} \wedge \omega,
$$

where $\omega$ is the unit volume form on $S^{3}$ and $F_{4}$ is an integral four-form on $X^{6}$, which is dual to an integral two cycle class $Q \in H_{2}\left(X^{6}, \mathbf{Z}\right)$. The setup is very similar to the main setup of this paper where we can view this as a circle compactification of the Euclidean 5d black hole geometry $X^{6} \times S^{3} \times A d S_{2}$. This is holographically dual to computation of the black holes corresponding to $M 2$ branes wrapping 2-cycles of $X^{6}$. Just as in our main example, we can write the mini-superspace wave function. In this case the mini-superspace will involve Kähler structure of $X^{6}$ which we denote by $k$ and flux through $S^{3}$ which we denote by $F_{4}$. It will not depend on the complex moduli of Calabi-Yau, nor on the radius of $S^{1}$. A reasoning very similar to our discussion for $4 \mathrm{~d}$ black holes leads us to

$$
\Psi\left(k, F_{4}\right)=\exp \left(-\frac{\pi}{12} \int_{X^{6}} k \wedge k \wedge k+\frac{\pi}{2} \int_{X^{6}} k \wedge F_{4}\right) .
$$

This was motivated as follows: As discussed before, by holography this wave function should have the property that

$$
\Omega(Q)=\int d k\left|\Psi\left(k, F_{4}\right)\right|^{2},
$$

and by the recent observation in [25] the leading saddle point computation gives

$$
\Omega(Q)=\exp \left(\frac{\pi \sqrt{2}}{3} \int_{X^{6}} F_{4}^{\frac{3}{2}}\right),
$$

which indeed agrees with the semi-classical expectation for the entropy. Moreover the wave function is peaked at

$$
\frac{1}{2} k \wedge k=F_{4}
$$

which is the attractor equation for $5 \mathrm{~d}$ black hole, fixing the Kähler moduli in terms of the flux.

The above wave function is expected to be true to all orders in the $1 / N$ expansion, but not beyond that, just as in the 4d black hole case discussed in this paper. It also suggests that the entropy as a function of charge $Q$ is an Airy function, to all orders in the $1 / Q$ expansion. It would be interesting to check this. 


\section{Applications}

We will end this paper with discussion on some directions for future research.

\subsection{Connection with Cosmology: Attaching to Lorentzian Signature Spacetime?}

The purpose of the original Hartle-Hawking construction of the wavefunction is to use it to set up an initial condition for a universe with a positive cosmological [2]. In the previous sections, we argued that the topological string partition function gives an analogous wave-function when the cosmological constant is negative. Since the Hilbert space is common between Lorentzian and Euclidean signature, we can view our state $|\psi\rangle$ as a state in the Lorentzian signature mini-superspace Hilbert space. It would then be natural to evolve this in time. This is, however, not as trivial as it my appear. The reason for this is that the Euclidean signature metric on $H_{2} / \mathbf{Z}$ is given by

$$
d s_{E}^{2}=d \rho^{2}+e^{2 \rho} d \tau^{2}
$$

where $\tau$ is periodically identified as $\tau \sim \tau+\beta$. In the previous section, we regarded $\rho$ as the Euclidean time of this geometry and constructed the wavefunction $\Psi$. If we cut the Euclidean geometry at some value of $\rho$, and attempt to glue a Minkowski solution by analytic continuation $\rho=i t$ the metric would not be real. Nevertheless it is clear that we have a well defined state in the Hilbert space of the Minkowski theory in the sense of mini-superspace, and thus it should give rise to some evolution in Lorentzian signature. This issue is currently under investigation.

\subsection{Statistical Interpretation of Topological String Wave-Function}

We have found a wave function for the mini-superspace in the context of flux compactification of type IIB on $S^{1} \times S^{2} \times M$, where the mini-superspace consists of the choice of flux $(p, q)$ as well as the extended complex moduli of $M$ (which includes the overall radius of $S^{2}$ ). We can use the wave function $\Psi_{p, q}(x)$ to measure the probability distribution for various fluxes and moduli. Note that we can compare the different fluxes here, as explained in section 3, because finite action instantons do interpolate between different flux sectors. 
From this result and the fact that the norm of the wave function is the exponential of the entropy, we are immediately led to the conclusion that the large fluxes, leading to more entropy, are most likely. In other words the flat space, corresponding to $p, q \rightarrow \infty$ is the most likely situation. Thus in this class of supersymmetric theories we would predict that the most likely situation is for the universe to be flat. This is very much in the spirit of Hartle-Hawking [2]. Note that this prediction is very different from the prediction one would have made based on counting the number of classical solutions. That would have led to equal probability for each flux $(p, q)$, as there is exactly one solution for each choice of flux.

We could also have asked the following question: Suppose we know that flux is a given value $(p, q)$, i.e. that has been already measured. Then what is the probability of finding various $X^{I}$ ? Then we can use the wave function in that sector and the conclusion would be that it is peaked at the attractor value, as already discussed. We can ask how strongly peaked is it? From the leading part of the wave function (including the measure) which is

$$
\exp \left|X-X_{p, q}\right|^{2}
$$

we can conclude that the standard deviation away from the attractor value is of order 1, i.e.,

$$
\Delta X^{I} \sim 1
$$

This leads to the statement that the deviation of the Calabi-Yau moduli away from the attractor value is given by

$$
\frac{|\Delta X|}{|X|} \sim \frac{1}{Q}
$$

where $Q$ denotes the overall scale of the charge. For small charge $Q$, the moduli of Calabi-Yau is not very peaked near the attractor value; however for large $Q$ it becomes increasingly more peaked. This is consistent with the fact that for large $Q$ the radius of $S^{2}$ scales as $Q$ and so we end up in non-compact space in that limit, which should freeze the moduli of Calabi-Yau.

It is quite gratifying to see that we can compare various string compactification data if we compactify all spatial moduli. It would be very useful to 
find more such examples within string theory and move toward a more realistic quantum cosmology within string theory.

\section{Acknowledgments}

We would like to thank F. Denef, R. Dijkgraaf, G. Gibbons, R. Gopakumar, S. Gukov, G. Mandal, S. Minwalla, L. Motl, A. Neitzke, J. Preskill, A. Strominger, and L. Susskind for useful discussions.

The research of H.O. was supported in part by DOE grant DE-FG03-92ER40701. The research of C.V. was supported in part by NSF grants PHY0244821 and DMS-0244464. 


\section{References}

[1] H. Ooguri, A. Strominger, and C. Vafa, "Black hole attractors and the topological string," Phys. Rev. D70 (2004) 106007, hep-th/0405146.

[2] J. B. Hartle and S. W. Hawking, "Wave function of the universe," Phys. Rev. D 28 (1983) 2960.

[3] H. Firouzjahi, S. Sarangi, S.-H. Tye, "Spontaneous Creation of Inflationary Universes and the Cosmic Landscape", JHEP 0409, 060 (2004); hepth/0406107

[4] A. Kobakhidze and L. Mersini-Houghton, "Birth of the universe from the landscape of string theory," hep-th/0410213.

[5] S. Ferrara, R. Kallosh and A. Strominger, "N $\mathcal{N}=2$ extremal black holes", Phys. Rev. D52 512 (1995), hep-th/9508072.

[6] A. Strominger, "Macroscopic Entropy of $\mathcal{N}=2$ black holes," Phys. Lett. B383 (1996) 39, hep-th/9602111.

[7] S. Kachru, R. Kallosh, A. Linde and S. P. Trivedi, "De Sitter vacua in string theory," Phys. Rev. D 68, 046005 (2003); hep-th/0301240.

[8] S. Gukov, C. Vafa and E. Witten, "CFT's from Calabi-Yau four-folds," Nucl. Phys. B 584, 69 (2000), [Erratum-ibid. B 608, 477 (2001)]; hepth/9906070.

[9] S. B. Giddings, S. Kachru and J. Polchinski, "Hierarchies from fluxes in string compactifications," Phys. Rev. D 66, 106006 (2002); hepth/0105097.

[10] K. Behrndt, G. Lopes Cardoso, B. de Wit, R. Kallosh, D. Lüst and T. Mohaupt, "Classical and quantum $\mathcal{N}=2$ supersymmetric black holes", Nucl. Phys. B488, 236 (1997); hep-th/9610105

[11] S. Ferrara, G. W. Gibbons and R. Kallosh, "Black holes and critical points in moduli space," Nucl. Phys. B 500 (1997) 75; hep-th/9702103.

[12] F. Denef, "Supergravity flows and D-brane stability," JHEP 0008 (2000) 050; hep-th/0005049.

[13] S. Cecotti and C. Vafa, "Topological anti-topological fusion," Nucl. Phys. B 367 (1991) 359.

[14] K. Hori, A. Iqbal and C. Vafa, "D-branes and mirror symmetry," hepth/0005247.

[15] H. Goldstein, C. P. Poole, and J. L. Safko, "Classical Mechanics," (AddisonWesley, 2002).

[16] E. Witten, "Quantum background independence in string theory," hepth/9306122. 
[17] M. Bershadsky, S. Ceccoti, H. Ooguri and C. Vafa, "Kodaira-Spencer theory of gravity and exact results for quantum string amplitudes," Commun. Math. Phys. 165 (1994) 311, hep-th/9309140.

[18] G. Lopes Cardoso, B. de Wit and T. Mohaupt, "Macroscopic entropy formulae and non-holomorphic corrections for supersymmetric black holes," Nucl. Phys. B 567 (2000) 87; hep-th/9906094.

[19] C. Vafa, "Two-dimensional Yang-Mills, black holes and topological strings," hep-th/0406058.

[20] M. Aganagic, H. Ooguri, N. Saulina and C. Vafa, "Black holes, qdeformed 2d Yang-Mills, and non-perturbative topological strings," hepth/0411280.

[21] A. Dabholkar, "Exact counting of black hole microstates," hep-th/0409148.

[22] A. Dabholkar, F. Denef, G. W. Moore and B. Pioline, "Exact and asymptotic degeneracies of small black holes," hep-th/0502157.

[23] E. Verlinde, "Attractors and the holomorphic anomaly," hep-th/0412139.

[24] R. Dijkgraaf, E. Verlinde and M. Vonk, "On the partition sum of the NS five-brane," hep-th/0205281.

[25] R. Dijkgraaf, S. Gukov, A. Neitzke, C. Vafa, "Topological M-theory as unification of form theories of gravity," hep-th/0411073. 